\title{
Time to hospital discharge and subsequent hospital readmission and mortality rates in ICU survivors
}

\author{
R Campbell', L Paton, P Stenhouse, A Mackay \\ From ESICM LIVES 2015 \\ Berlin, Germany. 3-7 October 2015
}

\section{Introduction}

As intensive care mortality falls, there is growing focus on the deficits in physical, mental and cognitive function encountered by survivors of critical illness. Termed the post-intensive care syndrome [1], this significantly and persistently impacts both health-related quality of life [2] and long term survival [3].

\section{Objectives}

We sought to review post-ICU outcomes in patients discharged from our unit to quantify the associated morbidity and mortality that exists following critical illness.

\section{Methods}

Patients surviving to ICU discharge at the Victoria Infirmary, Glasgow, between July 2012 and June 2013 were retrospectively identified and characterised using the WardWatcher ${ }^{\mathrm{TM}}$ database.

Subsequent hosptial admissions and mortality over a minimum of one year follow-up were determined using the Clinical Portal ${ }^{\mathrm{TM}}$ platform and data entered into an Excel $^{\mathrm{TM}}$ spreadsheet to facilitate analysis.

\section{Results}

During the study period, there were 240 admissions from 231 patients (121 men, 52.4\%) where survival to ICU discharge was achieved. This cohort had a mean age of 58 years (IQR $44-70)$ and mean APACHE score of 20 (IQR 14 - 25).

The Majority of ICU admissions were medical $(\mathrm{n}=143$, $61.9 \%)$ in comparison to surgical $(n=88,38.1 \%)$. Followup data indicated 199 patients (86.1\%) left hospital at a median of 11 days post-ICU discharge (IQR 5 - 19 days).

Over at least one year, 137 patients $(68.8 \%)$ were readmitted to hospital on 457 occasions and 24 (12.1\%) died.

There was a trend towards reduced survival and longer time to hospital discharge in older patients but no clear effect of age on subsequent hospital admission or mortality rates.

Table 1. Follow up data

\begin{tabular}{|c|c|c|c|c|}
\hline & $\begin{array}{l}\text { Survival to hospital } \\
\text { discharge }\end{array}$ & $\begin{array}{l}\text { Median time to hospital } \\
\text { discharge }\end{array}$ & $\begin{array}{l}\text { Subsequent hospital } \\
\text { admissions }\end{array}$ & $\begin{array}{l}\text { Subsequent } \\
\text { death }\end{array}$ \\
\hline $\begin{array}{l}\text { Whole cohort } \\
(n=231)\end{array}$ & 199 (86.1\%) & 11 days IQR [5-19] & $\begin{array}{l}137 \text { patients (68.8\%) } 457 \\
\text { admissions }\end{array}$ & 24 patients $(12.1 \%)$ \\
\hline$\leq 49$ years $(n=71)$ & $70(98.6 \%)$ & 6 days IQR [2-16] & $\begin{array}{l}44 \text { patients (62.9\%) } 163 \\
\text { admissions }\end{array}$ & 7 patients (10\%) \\
\hline$\geq 50$ years $(n=160)$ & $129(80.6 \%)$ & 12 days IQR [7-21] & $\begin{array}{l}93 \text { patients (72.1\%) } 294 \\
\text { admissions }\end{array}$ & 17 patients $(13.2 \%)$ \\
\hline$\geq 70$ years $(n=58)$ & $46(79.3 \%)$ & 17 days IQR [11.25-27.5] & 30 patients (65.2\%) 88 admissions & 8 patients $(13.7 \%)$ \\
\hline$\geq 80$ years $(n=21)$ & $15(71.4 \%)$ & 20 days IQR [14.5-27] & 10 patients (66.6\%) 26 admissions & 2 patients (13.3\%) \\
\hline
\end{tabular}




\section{Conclusions}

Our study demonstrates high hospital readmission and significant mortality rates among the proportion of critically ill patients surviving to hospital discharge.

Post intensive care syndrome is a major contributor to these adverse outcomes, but as yet, incompletely understood and inadequately addressed. Intensive rehabilitation may be needed if intensive care is to achieve more than survival, and deliver meaningful patient outcomes with appropriate resource utilisation.

Published: 1 October 2015

\section{References}

1. Needham DM, Davidson J, Cohen $\mathrm{H}_{\text {, et }}$ al: Improving long term outcomes after discharge from the intensive care unit. Critical Care Medicine 2012, 40:502-9.

2. Oeyen SG, Vandijck DM, Benoit DD, Annemans L, Decruyenaere JM: Quality of life after intensive care: a systematic review of the literature. Critical Care Medicine 2010, 38:2386-400.

3. Brinkman S, de Jonge E, Abu-Hanna A, Arbous MS, de Lange DW, de Keizer NF: Mortality after hospital discharge in ICU patients. Critical Care Medicine 2013, 41:1229-36.

doi:10.1186/2197-425X-3-S1-A763

Cite this article as: Campbell et al:: Time to hospital discharge and subsequent hospital readmission and mortality rates in ICU survivors. Intensive Care Medicine Experimental 2015 3(Suppl 1):A763.

\section{Submit your manuscript to a SpringerOpen ${ }^{\mathcal{O}}$ journal and benefit from:}

- Convenient online submission

- Rigorous peer review

- Immediate publication on acceptance

- Open access: articles freely available online

- High visibility within the field

- Retaining the copyright to your article 